\title{
Proposed mechanism for the formation of cold dense structures in plasmas
}

\author{
M. Z. Tokar and M. Koltunov \\ Institut für Energie und Klimaforschung_Plasmaphysik, Forschungszentrum Jülich, Association EURATOM-FZJ, \\ Trilateral Euregio Cluster, D-52425 Jülich, Germany
}

(Received 17 November 2011; revised manuscript received 16 February 2012; published 23 April 2012)

\begin{abstract}
The formation of cold dense structures in a hydrogen isotope plasma, caused by the penetration of impurities from a localized source, is modelled numerically. It is shown that a bubble structure with very high densities and low temperatures of all plasma components, i.e. electrons, main and impurity ions, arises if the density of impurity neutrals in the source exceeds a critical level. The major cause of this condensation phenomenon are Coulomb collisions between main and impurity ion species which, on the one hand, cool down the main ions and, on the other hand, confine impurity ions near the source. This mechanism is efficient even if the impurity radiation, considered usually as the most important cause of condensation instabilities, is completely switched off in the calculations. On the contrary, without ion-ion Coulomb collisions the radiation losses result only in a transient cooling of electrons with a later recovery of their temperature to a level comparable with the initial one.
\end{abstract}

DOI: 10.1103/PhysRevE.85.046412

PACS number(s): 52.35.Py, 52.55.Fa

\section{INTRODUCTION}

Compact structures with cold dense plasma often arise in fusion devices. A prominent example of these phenomena is the development of multifaceted radiation from the edge (MARFE) [1] close to the density limit [2], manifesting itself as a toroidally symmetric loop of cold, dense, and luminous plasma at the inner edge. Instabilities caused by impurity radiation, originally considered as a possible cause for the star formation from a primordial homogeneous interstellar medium [3-5], are usually suspected as a MARFE trigger [6,7]. The mechanism of such an instability is simple: A spontaneous reduction of the temperature in some region leads to a local drop of the pressure there, thus particles flow into this region; consequently, the plasma density increases, radiation losses grow, and the original temperature perturbation is enhanced. The usage of deliberate impurity injection, in particular for disruption mitigation purposes [8], has revealed other mechanisms of impurity influence, especially the cooling of the main plasma components in Coulomb collisions with cold impurity ions [9]. Therefore, it is enlightening to examine the relevance of these mechanisms to the formation of compact cold dense structures. Since the MARFE development is essentially affected by such processes as the loss of charged particles to the machine walls and ionization of recycling hydrogen neutrals [10], we consider a simpler situation with impurity injection into a homogeneous plasma without contact with material walls. This situation is obviously idealized, but it catches important facets of experiments with puffing of gaseous impurities into a tokamak [11,12].

Usually radiating impurity particles enter the plasma as neutrals with a temperature significantly lower than those of background plasma components. Coulomb collisions with impurity ions, generated by the ionization of neutrals, provide an effective channel to reduce the temperature of the plasma's main ions. This offers an independent self-sustaining mechanism for the structure formation since the collision frequency increases nonlinearly, as $T_{i}^{-1.5}$, with decreasing ion temperature. The friction between main and impurity ions, induced by collisions, counteracts the forces dispersing impurity ions from their source. These forces arise due to the impurity pressure gradient and electric field. The former comes up since there is a maximum in the impurity ion density at the source center, the latter because, owing to plasma quasineutrality, the electron density also has a maximum there. Upon reducing $T_{i}$ and increasing friction the impurity cloud shrinks, the impurity ion density grows, collisions are intensified, and $T_{i}$ drops further. Also the radiation energy losses increase, further decreasing the electron temperature. As a result a region with high densities and low temperatures of all plasma components arises. In the present paper we demonstrate that this, however, happens if the impurity neutral density exceeds some critical level. Below this level the forces, dispersing impurity ions, win the competition. Our calculations show that this scenario is effective even if the radiation losses are completely switched off but not vice versa.

\section{BASIC EQUATIONS}

Impurity species are released in fusion devices by plasmawall interactions or seeded deliberately for diverse purposes [11-15]. They enter the plasma as neutrals and normally their influxes are strongly concentrated at some specific locations. By ionization of neutrals with electrons, singly charged impurity ions are produced and spread out along the magnetic field. They are accelerated by their own pressure gradient and parallel electric field, but are then braked and heated up in Coulomb collisions with the main ions. Finally they disappear, being ionized into higher charge states. The impact of these stronger charged ions on local plasma behavior near the impurity injection position is neglected henceforth. This approximation is relevant for helium and lithium impurities. Both elements are used in fusion for diagnostic purposes; in addition, $\mathrm{He}$ is a product of thermonuclear reactions and $\mathrm{Li}$ is utilized as a plasma facing material. The doubly charged $\mathrm{He}$ ions are completely stripped and spread out over the whole magnetic surfaces. The ionization energy of $\mathrm{Li}^{2+}$ ions exceeds $100 \mathrm{eV}$, so at the plasma edge where the electron temperature is several tens of electron volts they disappear very slowly and have enough time for spreading out. Furthermore, even for other impurities, e.g., argon, ions with charges above one start to affect the local plasma visibly at injection rates unrealistically high for many applications (see Ref. [16]). The 
transport across the magnetic field is modeled as a diffusion in the direction $x$ with the diffusivity $D$.

Time evolution of the density of singly charged impurity ions $n_{1}$ is governed by a continuity equation [17]

$$
\frac{\partial n_{1}}{\partial t}-\frac{\partial}{\partial x}\left(D \frac{\partial n_{1}}{\partial x}\right)+\frac{\partial \Gamma_{1}}{\partial z}=n_{0} n_{e} k_{\mathrm{ion}}^{0}-n_{1} n_{e} k_{\mathrm{ion}}^{1},
$$

where $\Gamma_{1}$ is the density of the impurity ion flux in the magnetic field direction $z, n_{0}$ is the density of impurity neutrals, $n_{e}$ is the electron density, and $k_{\text {ion }}^{0}$ and $k_{\text {ion }}^{1}$ are the ionization rate coefficients. Due to plasma quasineutrality

$$
n_{e} \approx n_{i}+n_{1},
$$

with $n_{i}$ being the density of the main ions. The behavior of $\Gamma_{1}$ is governed by the momentum balance equation

$$
\begin{aligned}
& m \frac{\partial \Gamma_{1}}{\partial t}-m \frac{\partial}{\partial x}\left(D \frac{\partial n_{1}}{\partial x} \frac{\Gamma_{1}}{n_{1}}\right)+m \frac{\partial}{\partial z}\left(\frac{\Gamma_{1}^{2}}{n_{1}}\right) \\
& =-m\left(k_{\text {ion }}^{1} n_{e}+k_{i 1} n_{i}\right) \Gamma_{1}-\frac{\partial\left(n_{1} T_{1}\right)}{\partial z}+e E_{z} n_{1} .
\end{aligned}
$$

Here $m$ is the impurity particle mass and $k_{i 1} \sim T_{i}^{-1.5} m_{i}^{-0.5}$ is the rate coefficient characterizing the friction of impurity ions by Coulomb collisions with the background ions of the mass $m_{i}$ and temperature $T_{i}$. In the pressure gradient, the second term on the right-hand side (rhs) of Eq. (3), $T_{1}$ is the impurity ion temperature controlled by the heat balance equation

$$
\begin{aligned}
\frac{3}{2} n_{1} \frac{\partial T_{1}}{\partial t}+T_{1} \frac{\partial \Gamma_{1}}{\partial z}= & \frac{3}{2}\left(T_{0}-T_{1}\right) n_{0} n_{e} k_{\text {ion }}^{0} \\
& +3 n_{1}\left[n_{i} k_{i 1}\left(T_{i}-T_{1}\right)+n_{e} k_{e 1}\left(T_{e}-T_{1}\right)\right],
\end{aligned}
$$

where $T_{0}$ and $T_{e}$ are the temperatures of impurity neutrals and electrons, respectively, and $k_{e 1}$ is the rate coefficient for Coulomb collisions of impurity ions with electrons. The last term on the rhs of Eq. (3) is the force due to the electric field. Its component along the magnetic field $E_{z}$ arises in response to the parallel gradient of the electron pressure; by neglecting the inertia of electrons,

$$
e n_{e} E_{z}=-\partial\left(n_{e} T_{e}\right) / \partial z .
$$

Radiation losses and Coulomb collisions with impurities cool down the main plasma components and their temperatures are governed by heat balance equations. These take into account the heat conduction, heat exchange by Coulomb collisions, and energy losses from electrons by ionization and excitation of impurity particles:

$$
\begin{aligned}
& \frac{3 n_{i}}{2} \frac{\partial T_{i}}{\partial t}+T_{i} \frac{\partial \Gamma_{i}}{\partial z}-\frac{\partial}{\partial x}\left(\kappa_{i \perp} \frac{\partial T_{i}}{\partial x}\right)-\frac{\partial}{\partial z}\left(\kappa_{i \|} \frac{\partial T_{i}}{\partial z}\right) \\
& =3 n_{i}\left[k_{e i} n_{e}\left(T_{e}-T_{i}\right)+k_{i 1} n_{1}\left(T_{1}-T_{i}\right)\right], \\
& \frac{3 n_{e}}{2} \frac{\partial T_{e}}{\partial t}+T_{e} \frac{\partial \Gamma_{e}}{\partial z}-\frac{\partial}{\partial x}\left(\kappa_{e \perp} \frac{\partial T_{e}}{\partial x}\right)-\frac{\partial}{\partial z}\left(\kappa_{e \|} \frac{\partial T_{e}}{\partial z}\right) \\
& =n_{e}\left\{3 n_{i} k_{e i}\left(T_{i}-T_{e}\right)+3 n_{1} k_{e 1}\left(T_{1}-T_{e}\right)\right. \\
& \left.\quad-\sum_{j=0,1} n_{j}\left[k_{\text {ion }}^{j}\left(I_{j}+\frac{3}{2} T_{e}\right)+L_{j}\right]\right\}
\end{aligned}
$$

where $\Gamma_{i}$ and $\Gamma_{e}$ are the densities of the main ion and electron fluxes along the magnetic field; $\kappa_{e \perp}, \kappa_{i \perp}, \kappa_{e \|}$, and $\kappa_{i \|}$ are the components of the heat conduction coefficients, $k_{e i}$ accounts for Coulomb collisions between electrons and the main ions, and $I_{j}$ and $L_{j}$ are the ionization energy and cooling rate of impurity ions, respectively. The reduction of $T_{e}$ and $T_{i}$ leads to a parallel pressure gradient that generates a flow of the main ions toward the impurity source. An accompanying change of the main ion density is governed by the continuity equation

$$
\frac{\partial n_{i}}{\partial t}-\frac{\partial}{\partial x}\left(D \frac{\partial n_{i}}{\partial x}\right)+\frac{\partial \Gamma_{i}}{\partial z}=0,
$$

with $\Gamma_{i}$ determined by the main ion motion equation

$$
\begin{gathered}
m_{i} \frac{\partial \Gamma_{i}}{\partial t}-m_{i} \frac{\partial}{\partial x}\left(D \frac{\partial n_{i}}{\partial x} \frac{\Gamma_{i}}{n_{i}}\right)+m_{i} \frac{\partial}{\partial z}\left(\frac{\Gamma_{i}^{2}}{n_{i}}\right) \\
=-\frac{\partial\left(n_{i} T_{i}\right)}{\partial z}+e E_{z} n_{i} .
\end{gathered}
$$

The results in the present paper have been obtained by using Eq. (9) outside the cloud of singly charged impurity and therefore terms taking into account collisions between the main and impurity ions [see Eq. (3)], are omitted there.

In the initial state before impurity injection the densities and temperatures of electrons and main ions are assumed to be identical and homogeneous, $n_{e, i}(t=0, x, z)=n_{*}$ and $T_{e, i}(t=0, x, z)=T_{*}$. At $t=0$ the density of impurity neutrals is enhanced instantaneously in the source area $|x| \leqslant \delta_{0},|z| \leqslant$ $l_{0}$ to a prescribed level $n_{0}$ maintained constant afterward. The plasma parameters, which are perturbed in the vicinity of the impurity source, approach the values $n_{*}$ and $T_{*}$ far away from it. Equations (1)-(9) are nonlinearly strongly coupled partial differential equations. They are solved numerically, being reduced to a set of ordinary differential equations for the time evolution of several parameters, thus characterizing the solutions in principal details. This is done in the framework of the so-called shell model [16,18], where the whole space occupied by singly charged impurity ions is subdivided into the source $|x| \leqslant \delta_{0},|z| \leqslant l_{0}$ and decay zone $\delta_{0}<|x|, l_{0}<|l|$. In the latter the density $n_{1}$ decays to zero due to the ionization into the higher charge state. This behavior is formally reproduced with boundary conditions implying zero $\partial_{x} n_{1}, \partial_{z} n_{1}$, and $\Gamma_{1}$ at the source center position $x=z=0$ and $n_{1}, \Gamma_{1} \rightarrow 0$ far from the source $|x|,|z| \rightarrow \infty$. Approximate solutions of Eqs. (1) and (3) are presumed in the form $n_{1}(t, x, z)=n_{1}^{0}(t) \varphi(t, x) \psi(t, z)$ and $\Gamma_{1}(t, x, z)=\Gamma_{1}^{0}(t) \varphi(t, x) \chi(t, z)$. The functions $\varphi, \psi$, and $\chi$ are constructed by taking into account the boundary conditions above; the continuity of $n_{1}, \Gamma_{1}, \partial_{x} n_{1}, \partial_{z} n_{1}$, and $\partial_{x} \Gamma_{1}$; and the fact that $\Gamma_{1}$ approaches its maximum at the source boundary. Beyond the source $\varphi$ decays exponentially with some $e$-folding length $\delta_{d}$ and $\psi$ and $\chi$ decay with $l_{d}$. Figure 1 shows an example of the functions $\varphi, \psi$, and $\chi$ versus the dimensionless coordinate $\xi=x / \delta_{0}, z / l_{0}$ for some particular ratios $\delta_{d} / \delta_{0}$ and $l_{d} / l_{0}$. The time dependence of $\delta_{d}$ and $l_{d}$ introduces the time dependence of $\varphi, \psi$, and $\chi$. Equations of the shell model are obtained by integrating transport equations (1)-(9) over some subregions of the singly charged impurity ion shell (see Ref. [16]). These equations allow the computation of the time evolution of the parameters $n_{1}^{0}, \Gamma_{1}^{0}, \delta_{d}$, and $l_{d}$ and the characteristic parameters of the main plasma components $T_{e}$, 


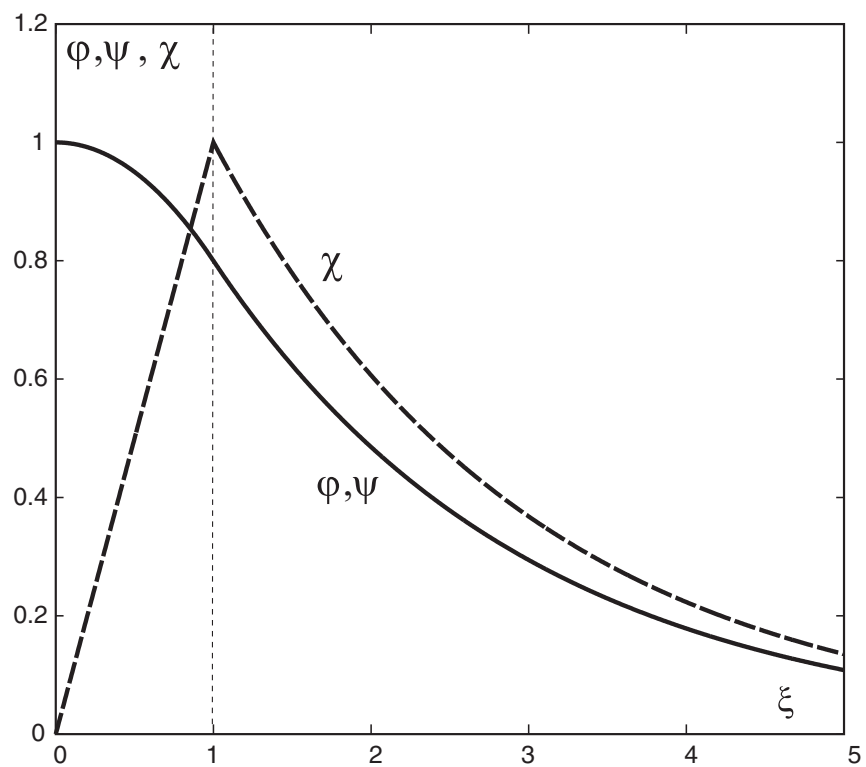

FIG. 1. Functions $\varphi\left(\xi \equiv x / \delta_{0}\right), \psi\left(\xi \equiv z / l_{0}\right)$, and $\chi\left(\xi \equiv z / l_{0}\right)$ for $\delta_{d} / \delta_{0}=l_{d} / l_{0}=2$.

$T_{i}$, and $n_{i}$ in the shell. The description of the latter parameters is the main development in the present paper compared to Refs. $[16,18]$. The explicit derivation of the shell model equations for these parameters is not included herein.

\section{RESULTS OF CALCULATIONS}

Computations have been done for lithium impurity entering into a deuterium plasma with the initial parameters typical at the edge of a medium tokamak device, e.g., the tokamak experiment for technology oriented research [14]: $n_{*}=10^{19} \mathrm{~m}^{-3}$ and $T_{*}=50 \mathrm{eV}$. Figures 2 and 3 show the time evolution of the parameters in the shell of $\mathrm{Li}^{+}$ions. Computations were done for two close magnitudes of the density of Li neutral atoms with the temperature $T_{0}=0.03 \mathrm{eV}$, localized in the source with dimensions $\delta_{0}=l_{0}=0.01 \mathrm{~m}$; the perpendicular diffusivity of charged particles $D=1 \mathrm{~m}^{2} \mathrm{~s}^{-1}$. For $n_{0}=0.75 \times 10^{19} \mathrm{~m}^{-3}$ the initial transient cooling of electrons, mostly due to energy losses through ionization and radiation of impurity atoms, is not supported by the main ions. Finally, owing to heating of electron in collisions with the main ions, $T_{e}$ relaxes to a level comparable with the initial one. Both excitation of impurity ions and Coulomb collisions with them are not efficient enough to preserve electron cooling because $n_{1}$ is limited at a low level: In collisions with the main ions, the impurity ions are heated up and are dissolved by their pressure gradient and electric field on a distance of several meters along the magnetic field.

In the case of a slightly higher $n_{0}=10^{19} \mathrm{~m}^{-3}$ a compact bubble of very dense and cold plasma, with a dimension along the magnetic field of several centimeters, develops. Impurity ions are confined in this structure by the friction with the main ions and their density $n_{1}$ and thus $n_{e}$ exceeds the initial plasma density by two orders of magnitude. However, this state cannot be consistently described in the framework of the present model because it does not account for the recombination processes being important under such conditions.

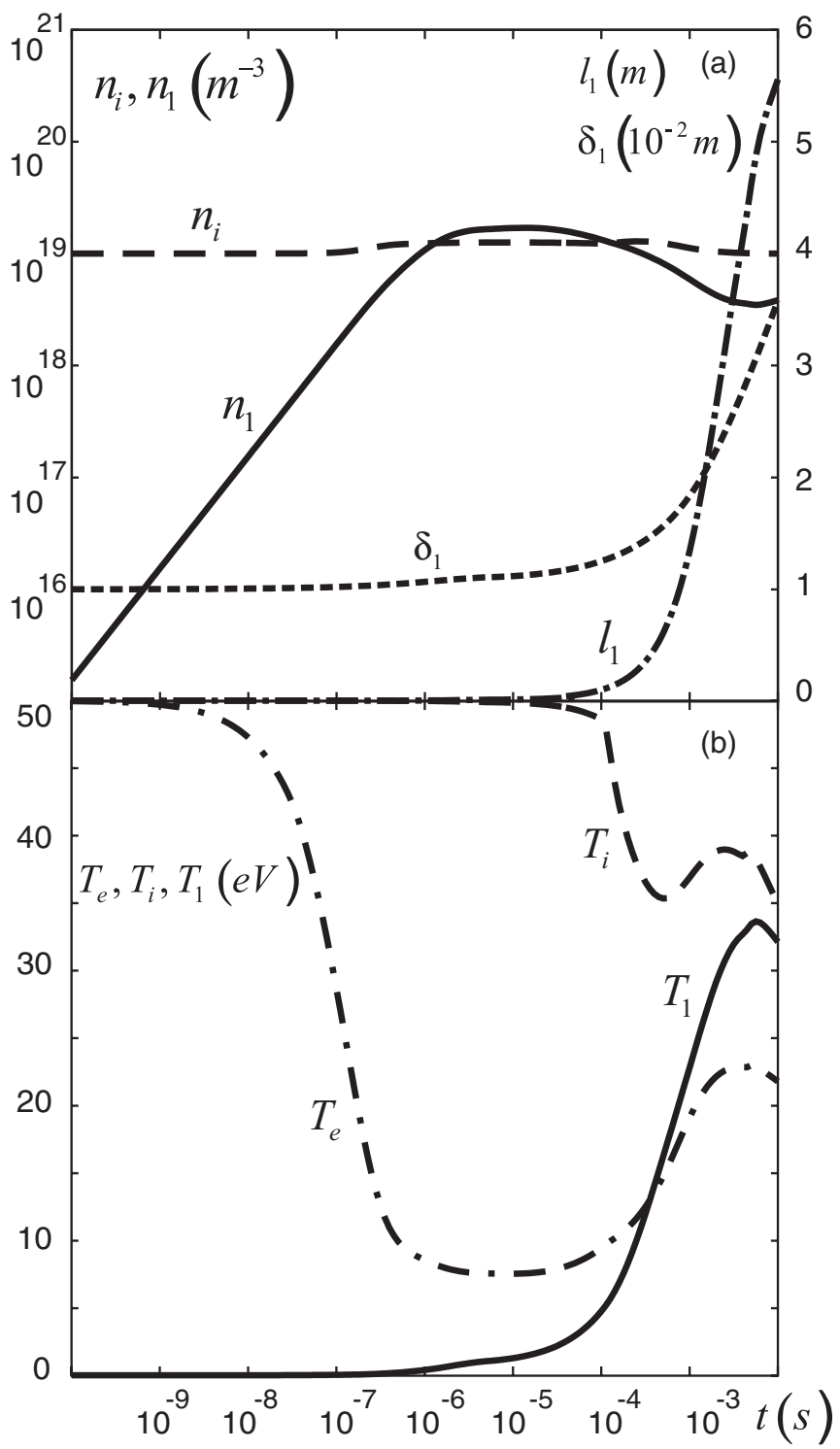

FIG. 2. Time evolution of (a) the densities of $\mathrm{Li}^{+}$ions $n_{1}$, deuterons $n_{i}$, and $\mathrm{Li}^{+}$-ion shell extensions perpendicular $\delta_{1} \equiv \delta_{0}+\delta_{d}$ and parallel $l_{1} \equiv l_{0}+l_{d}$ to the magnetic field and (b) the temperatures of $\mathrm{Li}^{+}$ions $T_{1}$, the main ions $T_{i}$, and electrons $T_{e}$ computed with the density of Li neutrals $n_{0}=0.75 \times 10^{19} \mathrm{~m}^{-3}$.

Normally condensation phenomena such as that demonstrated by Fig. 3 are thought to be triggered by electron energy losses on excitation of impurity and finally radiated away. Our present model includes, in addition to this loss channel, Coulomb collisions between different plasma components. This allows the analysis of which mechanisms are the most important for the condensation and structure development. Figure 4 displays the time evolution of parameters computed under different assumptions for $n_{0}=1.5 \times 10^{19} \mathrm{~m}^{-3}$ above the critical level. One can see that in all cases there is an initial significant cooling of electrons and the main ions. However, if Coulomb collisions between the main and impurity ions are turned off, both $T_{i}$ and $T_{e}$ recover significantly and a cloud of cold dense plasma does not arise. In the case with collisions but without radiation losses, the initial cooling stage is dragged on, but finally condensation develops. 


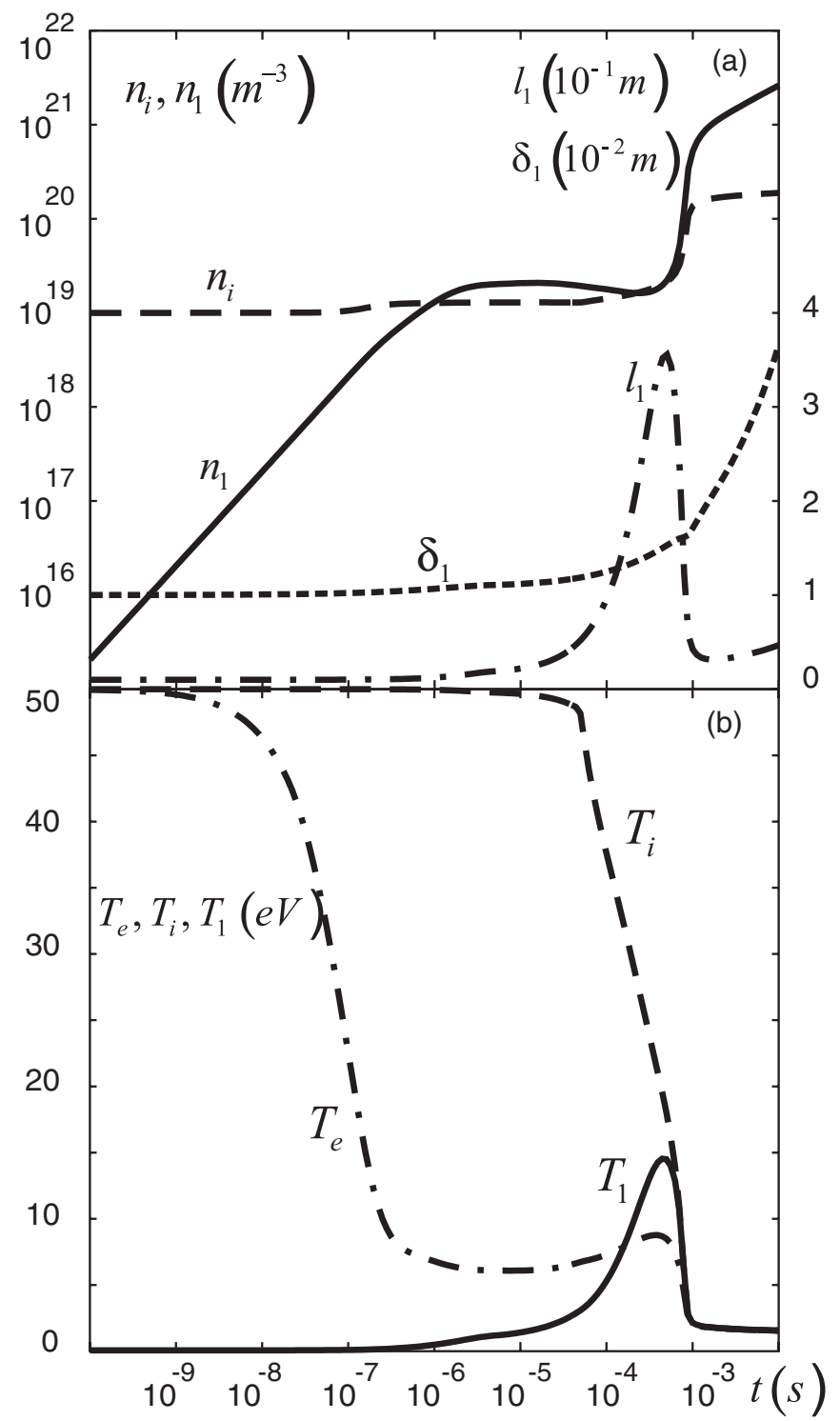

FIG. 3. Time evolution of (a) the densities of $\mathrm{Li}^{+}$ions $n_{1}$, deuterons $n_{i}$, and $\mathrm{Li}^{+}$-ion shell extensions perpendicular $\delta_{1}$ and parallel $l_{1}$ to the magnetic field and (b) the temperatures of $\mathrm{Li}^{+}$ions $T_{1}$, the main ions $T_{i}$, and electrons $T_{e}$ computed with the density of Li neutrals $n_{0}=10^{19} \mathrm{~m}^{-3}$.

Thus Coulomb collisions between ion components are vitally important for the formation of structures with cold dense plasma. Radiation losses alone can lead only to a transient drop in the electron temperature, recovering later to a level comparable with the unperturbed one. These findings offer a different look on condensation instabilities and structure formation triggered in hot plasmas by the presence of impurities. It differs principally from the standard explanation based on the effect of radiation losses $[3,4,6,7]$. Ion-ion Coulomb collisions reduce the temperature of the main ions and the collision frequency rises as $T_{i}^{-1.5}$. Impurity ions are confined better near the entrance position since their friction with the main ions counteracts impurity dispersion by the pressure gradient and electric field. The impurity ion density and collision frequency grow and $T_{i}$ drops further. This mechanism is enforced by the flow of the main ions toward

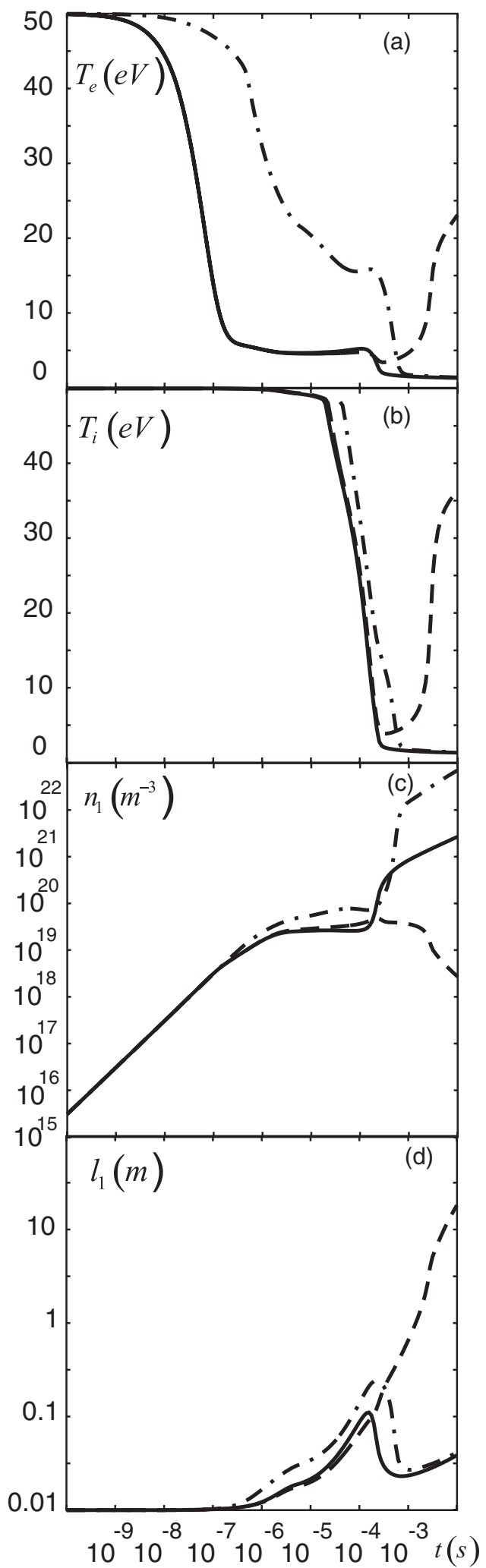

FIG. 4. Time evolution of the temperatures of (a) electrons and (b) deuterons, (c) the density of $\mathrm{Li}^{+}$impurity ions, and (d) the extension of the $\mathrm{Li}^{+}$- ion shell along the magnetic field computed for $n_{0}=1.5 \times 10^{19} \mathrm{~m}^{-3}$ with both Coulomb collisions between the main and impurity ions and radiation energy losses taken into account (solid curves), switched off collisions (dashed curves), and switched off radiation losses (dash-dotted curves). 
the impurity source. That is generated since the temperature reduction here results in a dropping plasma pressure. It has already been noticed for homogenous plasmas (see Ref. [9]) that collisions with heavier impurity ions are more efficient for cooling than impurity radiation. Here we show that the effect of ion-ion Coulomb collisions, operating through both cooling and friction, is of extreme importance also for the formation of compact cold and dense bubble structures.

We have stressed above that the formation of plasma structures discussed in this paper cannot be directly applied to the MARFE phenomenon. It is nonetheless instructive to analyze the relative importance of electron cooling through impurity radiation and cooling of the main ions by Coulomb collisions with impurities under the conditions typical for MARFE development. To make the results more transparent, constraints typical for a radiation instability analysis are imposed. We neglect in Eqs. (6)-(9) the contributions from perpendicular transport, the energy loss on neutral radiation, the electron-ion heat exchange, and the contribution of impurity ions to the electron density, i.e., $n \equiv n_{e}=n_{i}$ is assumed henceforth. Instead of Eqs. (5) and (9) the pressure constancy along the magnetic field

$$
n\left(T_{e}+T_{i}\right)=\text { const. }
$$

is adopted. This follows from the sum of Eqs. (5) and (9) where nonstationary and nonlinear terms $\partial_{t} \Gamma_{i}$ and $\partial_{z}\left(\Gamma_{i}^{2} / n_{i}\right)$, respectively, are omitted. (The latter allows us to exclude acoustic waves, which inessential for the present consideration.) Assume that electron and ion temperatures, equal to $T_{*}$ in the initial stationary state, spontaneously get small perturbations $\tilde{T}_{e, i}=\hat{T}_{e, i} \exp (\gamma t-i k z)$ with a certain wave vector $k$ along the magnetic field. Under these assumptions and for cold impurity ions $T_{1} \ll T_{*}$, Eqs. (6) and (7) provide a system of equations for the initial perturbation amplitudes $\hat{T}_{e, i}$ :

$$
\begin{array}{r}
\gamma\left(2 \hat{T}_{i}+0.5 \hat{T}_{e}\right)+\frac{k^{2} \kappa_{i \|}}{n} \hat{T}_{i}=\frac{3 m_{i} k_{i 1} n_{1}}{m}\left(\hat{T}_{i}+0.5 \hat{T}_{e}\right) \\
\gamma\left(2 \hat{T}_{e}+0.5 \hat{T}_{i}\right)+\frac{k^{2} \kappa_{e} \|}{n} \hat{T}_{e} \\
=n_{1}\left[\left(\frac{L_{1}}{2 T_{*}}-\frac{d L_{1}}{d T_{*}}\right) \hat{T}_{e}+\frac{L_{1}}{2 T_{*}} \hat{T}_{i}\right],
\end{array}
$$

The requirement for the existence of nontrivial solutions provides an equation for the perturbation growth rate $\gamma$. Consider two limit cases: (i) Collision ion cooling is dominant, i.e., $\hat{T}_{e} \ll \hat{T}_{i}$, and (ii) radiation electron cooling prevails, i.e., $\hat{T}_{i} \ll \hat{T}_{e}$. In the former Eq. (10) results in

$$
\gamma=\frac{3 m_{i}}{2 m} k_{i 1} n_{1}-\frac{k^{2} \kappa_{i \|}}{n}
$$

and in the latter Eq. (11) leads to

$$
\gamma=\left(\frac{L_{1}}{2 T_{*}}-\frac{d L_{1}}{d T_{*}}\right) \frac{n_{1}}{2}-\frac{k^{2} \kappa_{e \|}}{2 n},
$$

the simplest expression for the increment of radiation instability that is known from the literature. The condition for the instability threshold $\gamma=0$ provides a critical level of the parameter $\eta=n_{1} n / k^{2}$. For an ion-collision cooling instability

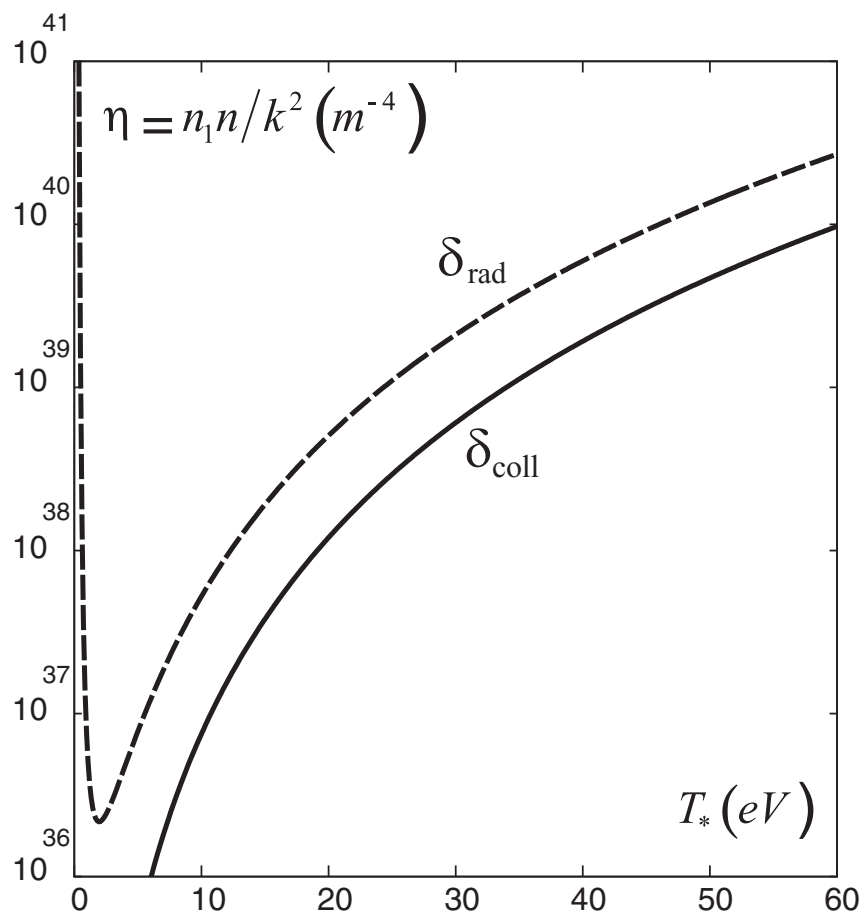

FIG. 5. Critical parameter $\eta$ for the development of thermal instabilities due to electron cooling with carbon impurity radiation and cooling of the main ions by Coulomb collisions with impurities.

this is

$$
\eta_{\mathrm{coll}}=\frac{m \kappa_{i \|}}{3 m_{i} k_{i 1}}
$$

and for an electron radiation cooling instability

$$
\eta_{\mathrm{rad}}=\frac{\kappa_{e \|}}{0.5 L_{1} / T_{*}-d L_{1} / d T_{*}} .
$$

For certain background plasma and impurity species $\eta_{\text {coll }}$ and $\eta_{\mathrm{rad}}$ are functions of the temperature $T_{*}$ only. Figure 5 demonstrates these dependences for carbon impurity in a deuterium plasma, a typical situation for tokamak devices [6], found with $L_{1}$ computed by using data from Ref. [19]. One can see that for the conditions usual for MARFE development, with the edge temperature below $50 \mathrm{eV}$, an ion-collision instability develops at $\eta$ at least several times smaller than the critical value for electron radiation instability. Thus, also by studying the MARFE development one has to take ion-ion collisions into account. For impurity ions with the charge $j$ higher than one the cooling rate does not differ significantly from $L_{1}$ but the friction coefficient increases as $j^{2}$. Therefore the conclusion above holds if impurity ions of higher charges are taken into account.

\section{CONCLUSION}

Impurities play a crucial role in hot fusion plasmas for the formation of compact structures where high densities and low temperature are achieved. Although impurity radiation has been considered already for a long time as a possible trigger of instabilities leading to the formation of structures, cooling of the plasma ions in Coulomb collisions with impurity 
ions has only been considered recently. In the present paper we demonstrate that the synergy of this cooling channel with the friction due to collisions, confining impurity particles in the vicinity of their source, can be more effective for structure generation that radiation energy losses. For the case of a singly charged lithium ion spreading in a tokamak deuterium plasma calculations demonstrate that structures arise if both friction and cooling due to ion-ion collisions are taken into consideration. Without these effects the energy losses from electrons on excitation and radiation of impurities lead to a transient plasma cooling only. Although the results obtained cannot be directly applied to MARFE formation in tokamaks, the estimates done show that for this phenomenon the ion cooling by Coulomb collisions can be more efficient than the electron cooling with radiation.

\section{ACKNOWLEDGMENT}

This work was partly supported by the German Research Foundation through the Research Training Group 1203.
[1] J. L. Terry, E. S. Marmar, S. M. Wolfe, D. Gwinn, M. Pickrell, B. Lipschultz, and M. Foord, Bull. Am. Phys. Soc. 26, 886 (1981).

[2] M. Greenwald, Plasma Phys. Control. Fusion 44, R27 (2002).

[3] E. N. Parker, Astrophys. J. 117, 431 (1953).

[4] G. B. Field, Astrophys. J. 142, 531 (1965).

[5] A. Brandenburg, M. J. Korpi, and A. J. Mee, Astrophys. J. 654, 945 (2007).

[6] B. Lipschultz, B. LaBombard, E. S. Marmar, M. M. Pickrell, J. L. Terry, R. Watterson, and S. M. Wolfe, Nucl. Fusion 24, 977 (1984).

[7] J. F. Drake, Phys. Fluids 30, 2429 (1987).

[8] R. S. Granetz, E. M. Hollmann, D. G. Whyte, V. A. Izzo, G. Y. Antar, A. Bader, M. Bakhtiari, T. Biewer, J. A. Boedo, T. E. Evans, I. H. Hutchinson, T. C. Jernigan, D. S. Gray, M. Groth, D. A. Humphreys, C. J. Lasnier, R. A. Moyer, P. B. Parks, M. L. Reinke, D. L. Rudakov, E. J. Strait, J. L. Terry, J. Wesley, W. P. West, G. Wurden, and J. Yu, Nucl. Fusion 47, 1086 (2007).

[9] E. O. Baronova and V. V. Vikhrev, Contrib. Plasma Phys. 50, 313 (2010).

[10] M. Z. Tokar, J. Rapp, D. Reiser, U. Samm, F. C. Schüller, G. Sergienko, and P. C. de Vries, J. Nucl. Mater. 266-269, 958 (1999).
[11] G. M. McCracken, U. Samm, B. Bertschinger, V. Philipps, R. A. Pitts, D. H. J. Goodall, S. J. Davies, G. van Oost, A. Pospieszczyk, R. Schorn, B. Schweer, P. C. Stangeby, G. Telesca, and G. Waidmann, J. Nucl. Mater. 196-198, 199 (1992).

[12] P. Wienhold, H. G. Esser, D. Hildebrandt, A. Kirschner, M. Mayer, V. Philipps, and M. Rubel, J. Nucl. Mater. 290-293, 362 (2001).

[13] K. McCormick, S. Fiedler, G. Kocsis, J. Schweinzer, and S. Zoletnik, Fusion Eng. Des. 34-35, 125 (1997).

[14] U. Samm, G. Bertschinger, P. Bogen, J. D. Hey, E. Hintz, L. Konen, Y. T. Lie, A. Pospieszczyk, D. Rusbuldt, R. P. Schorn, B. Schweer, M. Tokar, and B. Unterberg, Plasma Phys. Control. Fusion 35, B167 (1993).

[15] J. Ongena et al., Phys. Scr. 52, 449 (1995).

[16] M. Koltunov and M. Z. Tokar, Plasma Phys. Control. Fusion 54, 025003 (2012).

[17] P. C. Stangeby, The Plasma Boundary of Magnetic Fusion Devices (Institute of Physics, Bristol, 2000), pp. 277-357.

[18] M. Z. Tokar and M. Koltunov, in Numerical Realization of a Shell Model for Impurity Spreading in Plasmas, edited by T. E. Simos, G. Psihoyios, Ch. Tsitouras, and Z. Anastassi, AIP Conf. Proc. No. 1389 (AIP, New York, 2011), p. 1660.

[19] Y. Itikawa, S. Hara, T. Kato, S. Nakazaki, M. S. Pindzola, and D. H. Crandall, At. Data Nucl. Data Tables 33, 150 (1985). 